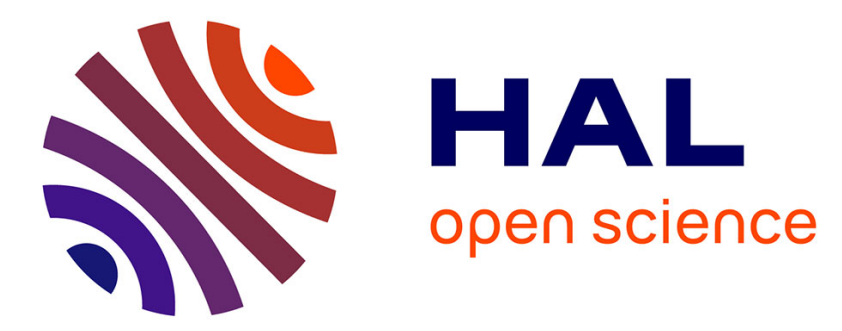

\title{
Magnetoresistance in magnetic nanoconstrictions: The role of structural defects Magnetoresistance in magnetic nanoconstrictions: The role of structural defects
}

Nicolas Biziere, M.-C Clochard, Pham Do, J.-E Wegrowe, M Viret

\section{- To cite this version:}

Nicolas Biziere, M.-C Clochard, Pham Do, J.-E Wegrowe, M Viret. Magnetoresistance in magnetic nanoconstrictions: The role of structural defects Magnetoresistance in magnetic nanoconstrictions: The role of structural defects. Journal of Applied Physics, 2013, pp.173910. 10.1063/1.4803702 . hal-01128122

\author{
HAL Id: hal-01128122 \\ https://hal.science/hal-01128122
}

Submitted on 9 Mar 2015

HAL is a multi-disciplinary open access archive for the deposit and dissemination of scientific research documents, whether they are published or not. The documents may come from teaching and research institutions in France or abroad, or from public or private research centers.
L'archive ouverte pluridisciplinaire HAL, est destinée au dépôt et à la diffusion de documents scientifiques de niveau recherche, publiés ou non, émanant des établissements d'enseignement et de recherche français ou étrangers, des laboratoires publics ou privés. 


\section{AIP $\mid \begin{aligned} & \text { Jumanol } \\ & \text { Applied Physics }\end{aligned}$}

\section{Magnetoresistance in magnetic nanoconstrictions: The role of structural defects}

N. Biziere, M.-C. Clochard, Pham Do Chung, J.-E. Wegrowe, and M. Viret

Citation: J. Appl. Phys. 113, 173910 (2013); doi: 10.1063/1.4803702

View online: http://dx.doi.org/10.1063/1.4803702

View Table of Contents: http://jap.aip.org/resource/1/JAPIAU/v113/i17

Published by the American Institute of Physics.

\section{Additional information on J. Appl. Phys.}

Journal Homepage: http://jap.aip.org/

Journal Information: http://jap.aip.org/about/about_the_journal

Top downloads: http://jap.aip.org/features/most_downloaded

Information for Authors: http://jap.aip.org/authors

\section{ADVERTISEMENT}

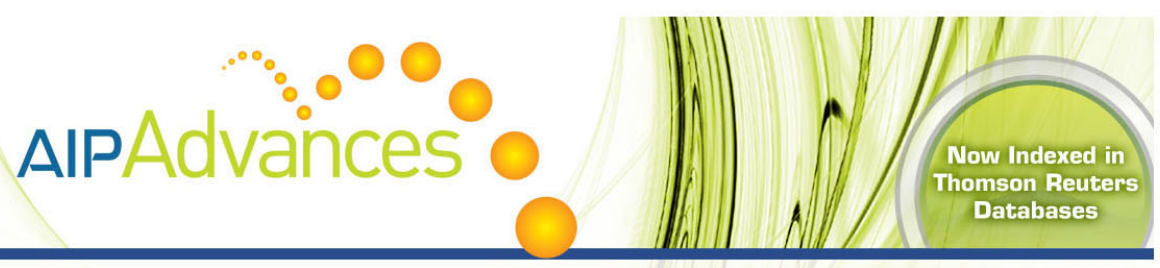

\section{Explore AIP's open access journal: Rapid publication \\ - Article-level metrics \\ - Post-publication rating and commenting}




\title{
Magnetoresistance in magnetic nanoconstrictions: The role of structural defects
}

\author{
N. Biziere, ${ }^{1}$ M.-C. Clochard,${ }^{2}$ Pham Do Chung, ${ }^{2}$ J.-E. Wegrowe, ${ }^{2, \text { a) }}$ and M. Viret ${ }^{3}$ \\ ${ }^{1}$ CNRS-CEMES, 29 rue Jeanne Marvig, 31055 Toulouse, France \\ ${ }^{2}$ Ecole Polytechnique, LSI, CNRS 7642 \& CEA/DSM/IRAMIS, Palaiseau F-91128, France \\ ${ }^{3}$ SPEC, CEA/DSM/IRAMIS \& URA CNRS 2464, 91191 Gif-Sur-Yvette Cedex, France
}

(Received 28 December 2012; accepted 16 April 2013; published online 7 May 2013)

\begin{abstract}
The resistance of a ferromagnetic cylindrical Ni nano-constriction has been measured as a function of the external magnetic field. Typical profiles of the resistance versus external field are observed with irreversible jumps separated by stable plateaus, showing systematic slow relaxation effects. An interpretation in terms of magnetomechanical strain at the level of the constriction is proposed. This scenario allows the slow relaxation behaviour to be understood in terms of displacement of dislocations or similar metastable structural defects. (C) 2013 AIP Publishing LLC.

[http://dx.doi.org/10.1063/1.4803702]
\end{abstract}

\section{INTRODUCTION}

In the frame of the recent development of a spin based electronics and the subsequent miniaturization of devices, it is of central interest to understand the behavior of domain walls (DWs) in magnetic nanostructures. Since the 1990s, a large research effort has been applied to studying how small constrictions can trap the DWs and influence their physical properties. In particular, it has been predicted ${ }^{1}$ and observed ${ }^{2}$ that in constrictions of sizes below that of relaxed domain wall widths, the DWs distort and become narrower. This has consequences on their transport properties as increased magnetization gradients enhance spin dependent scattering. ${ }^{3}$ Moreover, the effect of a large current density passing through DWs has attracted a lot of attention lately. Indeed, it has been shown that spin polarized electrons can transfer angular momentum to the DWs resulting in an applied pressure able to move DWs without magnetic fields. ${ }^{4,5}$ Ultimately, atomic sized DWs acquire new properties linked to the ballistic nature of transport through one or a few atoms, ${ }^{6}$ but these are hard to push out of such narrow constrictions. Above all, it appears that at very small (i.e., atomic) scale, the effect of local deformation can be overwhelming. Indeed, magnetically induced strain (i.e., magnetostriction) has been shown to greatly influence the resistance response in most geometries ${ }^{8-10}$ and has been coined to be responsible for many impressive results of the reported literature. In the present work, a small constriction of a couple of nanometers is performed in a biconical wire of $10 \mu \mathrm{m}$ length with a conicity of $28^{\circ}$. The wire is connected electrically and the resistance is measured as a function of the magnetic field, i.e., as a function of the magnetization states. The measurements suggest that the resistance profile as a function of the magnetic field is due to mechanical properties. A magnetomechanical strain can modify the lattice structures (typically via dislocations) at the level of the constriction. This study is a prolongation of previous

\footnotetext{
${ }^{a)}$ Electronic address: jean-eric.wegrowe@polytechnique.fr
}

measurements performed on larger constrictions, ${ }^{11}$ in which the different states of the domain wall have been identified. ${ }^{12}$

\section{SAMPLE FABRICATION}

Cylindrical magnetic wires are typically obtained using polymer films irradiated by heavy ions where damaged tracks can be revealed by chemical etching. Magnetic materials can subsequently be deposited in the holes (most of the time by electro-deposition) thus making long cylindrical wires of nanometer size. To reveal tracks by chemical etching, the track etch-rate $v_{t}$ should be at least 10 times higher than the bulk etch-rate $v_{b}$. When one wants to tune the geometry, the best is to play both on the polymer chemistry composition to monitor its behaviour towards hydrolysis $\left(v_{b}\right)$ and on the track damages by sensitization techniques such as UV lightening $\left(v_{t}\right)$. If the polymer sensitivity towards hydrolysis is high, then the bulk etch-rate becomes closer to the track etch rate and diameters vary between the surface and the interior of the polymer. By using free standing irradiated polymer membranes, it is possible to favour bi-conical structures (Fig. 1).

Recently, we have found an interesting alkyl fluorinated copolymer, the poly(VHF-co-TrFE), which can undergo double cone track etching by combining the action of a strong oxidant to create hydrolysable bridges and strong alkaline etchant to cut the bridges inducing polymer chain scissions. ${ }^{12}$ Figure 2 shows that constrictions in the nanoscale range cannot be resolved by FESEM (Fig. 2(b)) but these images can be extrapolated to evaluate constriction sizes (Fig. 2(b)) and conicities. The aperture of conical pores is followed both by $\mathrm{pH}$ and conductimetry experimental set up as already described. ${ }^{13,14}$ At the energy range of $10 \mathrm{MeV} / \mathrm{mau}$, all ion tracks constitute a continuous trail of excitation resulting to a constant value for the track etch-rate $v_{t}$. To assure a constant value for $v_{b}$, the best way is to avoid tracks overlap by irradiating at low fluences, generally $10^{6}$ ions $\mathrm{cm}^{-2}$.

For the study of domain wall states in very small constrictions, we use the electrodeposition technique to grow nickel within the poly (VDF-co-TrFE) copolymer 


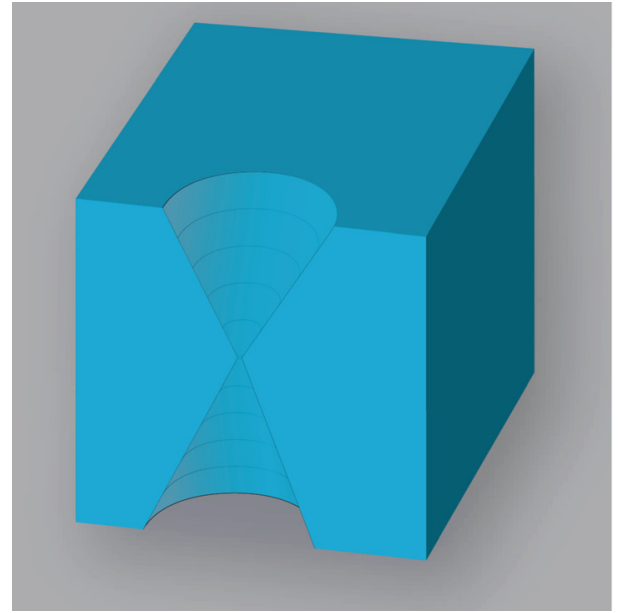

bulk etch rate

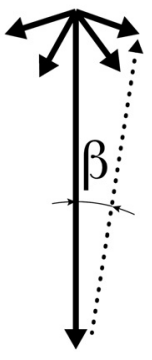

track etch rate

FIG. 1. Track etching considering a constant regime for $v_{t}$ and $v_{b}$ and $\sin \beta=v_{b} / v_{t}$.

membrane. We have chosen the sample presenting the wider conicity $\left(\beta=28^{\circ}\right)$ obtained for bi-conical shaped tracks with an estimated constriction size of $10 \pm 3 \mathrm{~nm}$ (Figure 2). A thick Au electrode is first deposited on one side of the membrane and is used as the working electrode. On the other side, a very thin gold layer (about $10 \mathrm{~nm}$ ) is sputtered to signal the stop of the electrodeposition and to contact the bi-conical nanowires. This procedure allows contacting only one or a few nanowires for electrical measurements. Details about the method are described elsewhere ${ }^{15,16}$ As the constriction is extremely small in a hydrophobic matrix, the electrodeposition time is long. At least $2000 \mathrm{~s}$ are necessary to fill the $10 \mu \mathrm{m}$ length of the biconical wire.

\section{MAGNETO-RESISTANCE MEASUREMENTS}

Magnetoresistance measurements have been performed as a function of the magnetic field, for a sample composed of
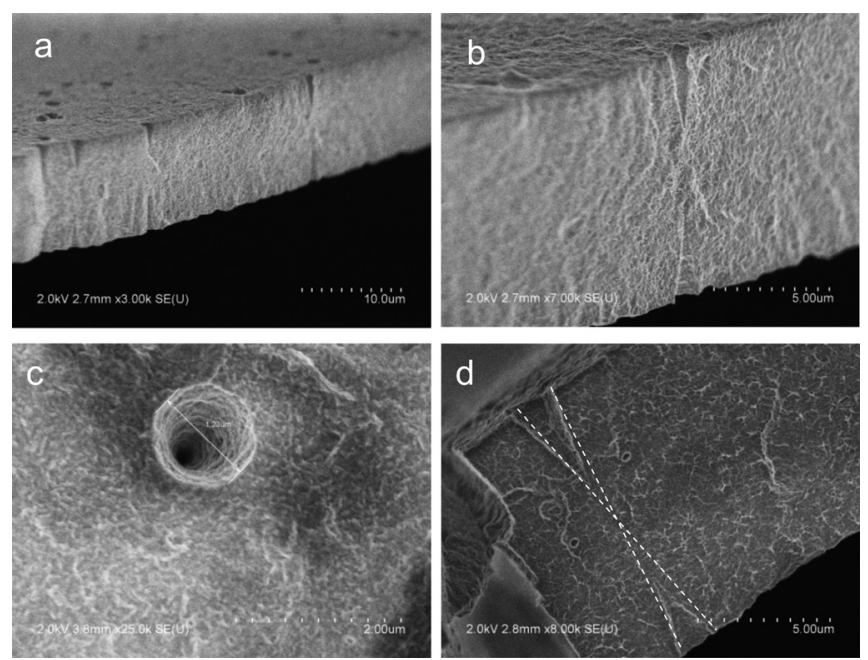

FIG. 2. FESEM images of bi-conical tracks obtained from etched poly(VDFco-TrFE) membranes irradiated with accelerated ${ }^{78} \mathrm{Kr}^{31+}(10 \mathrm{MeV} / \mathrm{mau})$ at a fluence of $10^{6}$ tracks $\mathrm{cm}^{-2}$ Etching conditions: $\mathrm{KOH} 10 \mathrm{~N} / \mathrm{KMnO}_{4} 0.25 \mathrm{~N}$ $80^{\circ} \mathrm{C}, 1 \mathrm{~h}$. (a) Global view of freeze-dried cut edge of an etched poly(VDFco-TrFE) membrane; (b) zoom of the cross-section focussed on a biconical track; (c) surface; and (d) extrapolation to determine the nanoconstriction size. a bundle of a few tens of Ni bicones contacted in parallel. The reproducibility from one sample to the other is rather poor, due to the fluctuations of the diameter of the constrictions, and due to the fact that the number of bicones contacted in parallel is too small to constitute a statistical ensemble (the thermodynamic limit is not reached). We focus here on a sample that shows the largest constriction effects.

The magnetization states are usually probed with anisotropic magnetoresistance (AMR), for which the electrical resistivity $\rho$ is due to inelastic electron scattering occurring in anisotropic ferromagnetic lattice. ${ }^{17}$ For $3 \mathrm{D}$ ferromagnets, the resistivity is large if the mean trajectory of the electrons is parallel to the magnetization, and weak if it is perpendicular to the magnetization. The electric resistivity is then related to the magnetization by the formula ${ }^{16,17}$

$$
\rho(\phi)=\rho_{0}+\Delta \rho \cos ^{2}(\phi),
$$

where $\phi$ is the angle between the local magnetization and the electric current density. In the case of cylindrical wires, the current is flowing along the wire axis (so that its density is homogeneous inside the wire) and the magnetization $M(H)$ is uniform. The relation Eq. (1) then becomes $R(H)=R_{0}$ $+\Delta R\left(M(H) / M_{s}\right)^{2}$, where $M_{s}$ is the magnetization at saturation. The amplitude $\Delta R$ of the AMR is comprised between $1 \%$ and $2 \%$ in usual $\mathrm{Ni}$ nanowires with cylindrical shape. Accordingly, the hysteresis loop of the resistance gives direct access to the hysteresis loop of the magnetization. In contrast, in the case of biconical nanowires, Eq. (1) is valid only locally (at the scale of few nanometers) since neither the current nor the magnetization is uniform. Numerical simulations are necessary in order to precisely describe the magnetic configurations measured through the magnetoresistance. ${ }^{11}$ Nevertheless, the profile $R(H)$ gives the mean value of the resistance averaged over a cone section, and the general shape follows approximately that of a uniformly magnetized wire, as shown in Fig. 3(a). Due to the biconical shape and the contact resistance, the amplitude of the AMR signal is small (typically one order of magnitude below that obtained for cylindrical wires).

According to a previous study performed on comparable samples with larger constrictions, ${ }^{11}$ the magnetization states can be roughly described as follows. At the remanent state, the magnetization within the constriction is almost uniform due to a strong shape anisotropy (see part II and III in Fig. 6 of Ref. 11). However, on each side of this uniformly magnetized domain, the conical shape of the wire leads to a vortex like state in the biggest part of the wire that decreases the magnetostatic energy of the system. This leads to particular dynamical switching process for which we observe pinning and depinning of vortex walls on each side of the constriction.

However, this corresponding AMR profile is not that measured if a narrow constriction is present inside one of the contacted bicones, as shown in Fig. 3(b). The magnetoresistance profile presented in Fig. 3(b) is measured in the same conditions as for Fig. 3(a)—with the external field perpendicular to the axis of the cone (angle $\theta=90^{\circ}$ ) —on a different sample. In the following, we focus the study exclusively on this last kind of samples. 


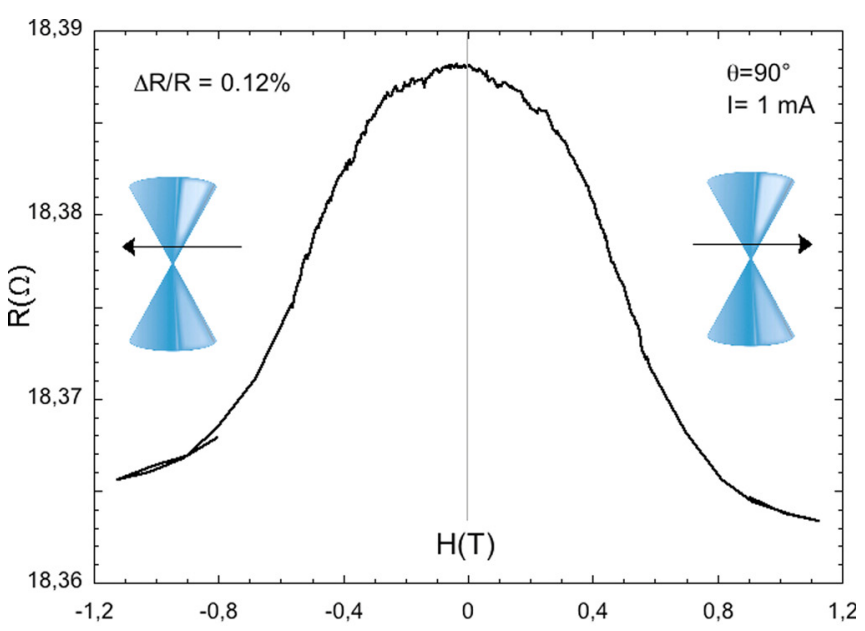

(a)

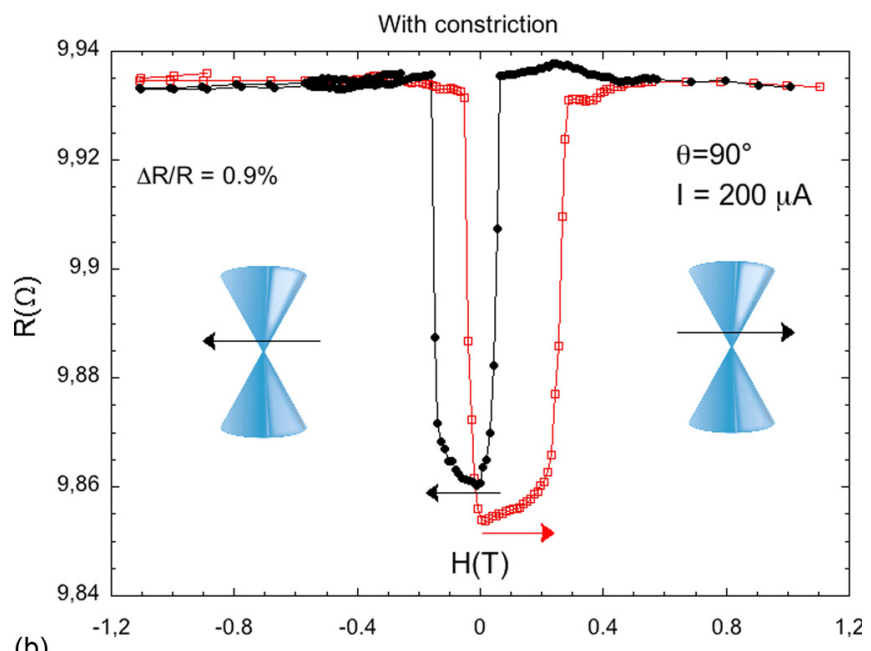

(b)

FIG. 3. Typical AMR profile measured with the external field perpendicular to the axis of the cone, at angle $\theta=90^{\circ}$. (a) Bicones with relatively large constrictions: the AMR profile is reversible. (b) Bicones with narrow constrictions: the magnetoresistance profile is hysteretic with positive magnetoresistance and saturation effects. Black dots: decreasing field, red square: increasing field.

As shown in Fig. 3(b), the general profile of the curve $R(H)$ is inverted with respect to the expected AMR profile, in the sense that the magnetic configuration perpendicular to the wire axis corresponds to high resistance. A hysteretic signal of typically $1 \%$ is observed in the region $+0.2 \mathrm{~T},-0.2 \mathrm{~T}$, which coincides with the region in which the magnetization is rotating from one direction to the other inside the cone.

Fig. 4 shows a typical hysteresis loop of the resistance at an intermediate angle of the applied field $\left(\theta=35^{\circ}\right.$ with respect to the cone axis) measured between 1.2 and $1.2 \mathrm{~T}$. As for the measurements at $\theta=90^{\circ}$, the hysteretic behavior (with a coercivity of about $0.1 \mathrm{~T}$ ) is restricted to the same field interval as that measured and studied in usual biconical samples. ${ }^{11}$ The main shape is reproducible while cycling the magnetic field, with some significant statistical fluctuations of the position of the jumps (6 superimposed hysteresis loops are shown in Fig. 4(b)). In contrast, the plateau corresponding to quasi-static states is well reproduced.

The most striking feature of this system consists in the systematic relaxation that occurs from unstable states to

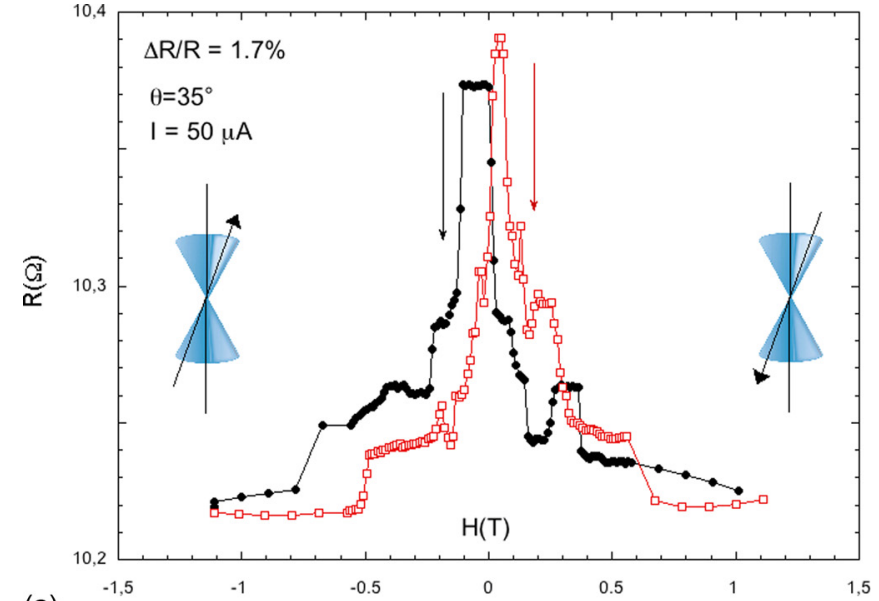

(a)

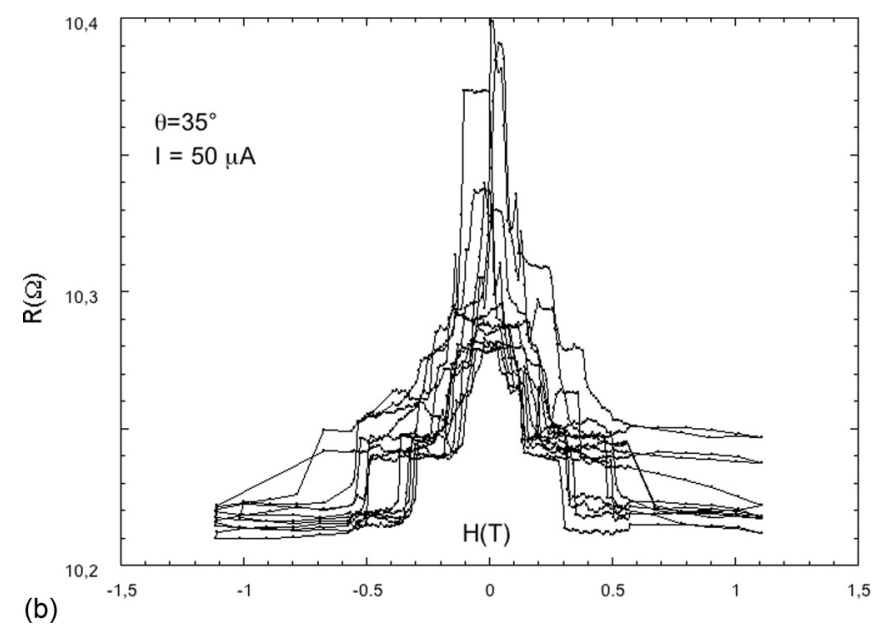

FIG. 4. Resistance as a function of the applied field. The applied field is at $\theta=35^{\circ}$ with respect to the cone axis. (a) The hysteresis loop is composed of irreversible jumps separated by reversible plateaus. (b) Reproducibility of the jumps and plateaus over six hysteresis loops.

more stable states. The relaxation mechanisms take different forms, from "instantaneous" jumps to slow relaxations and slow oscillations. Fig. 5 shows the time dependence of the hysteresis loops measured at small angle $\left(\theta=15^{\circ}\right)$ while cycling over ten consecutive $\pm 1.2 \mathrm{~T}$ loops. The system is relaxing slowly to more stable configurations while cycling the magnetic field (Fig. 5(a)). Three hysteresis loops are shown as a function of the applied field in Figs. 5(b)-5(d). The metastable nature of the corresponding magnetic states can be observed, including two level fluctuation effects (Fig. 5(d)).

The relaxation effects have been investigated at fixed field in different positions in the hysteresis loop. The saturation field of $-1.2 \mathrm{~T}$ is first applied. The field is thus rapidly switched off $(H=0 \mathrm{~T})$ before measuring the resistance as a function of time (Fig. 6). Note that the small variation of 0.1 $\Omega$ between the resistances measured in Figs. 5(b) and 6 is due to a thermal drift. An instantaneous jump is measured after a typical time (ranging from seconds to minutes) and is followed by a slow relaxation process, ending sometimes in an oscillatory regime without damping (inset of Fig. 6(a)). The periodicity of the oscillations is $T=920 \mathrm{~s}$ and the amplitude is $5 \mathrm{~m} \Omega$ (i.e., $\Delta R / R \approx 0.05 \%$ ). 
a)

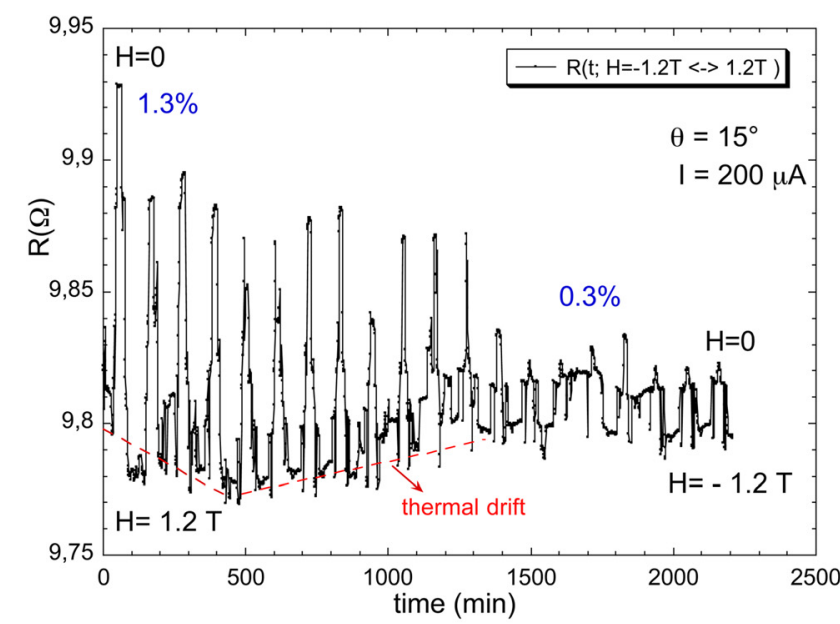

c)

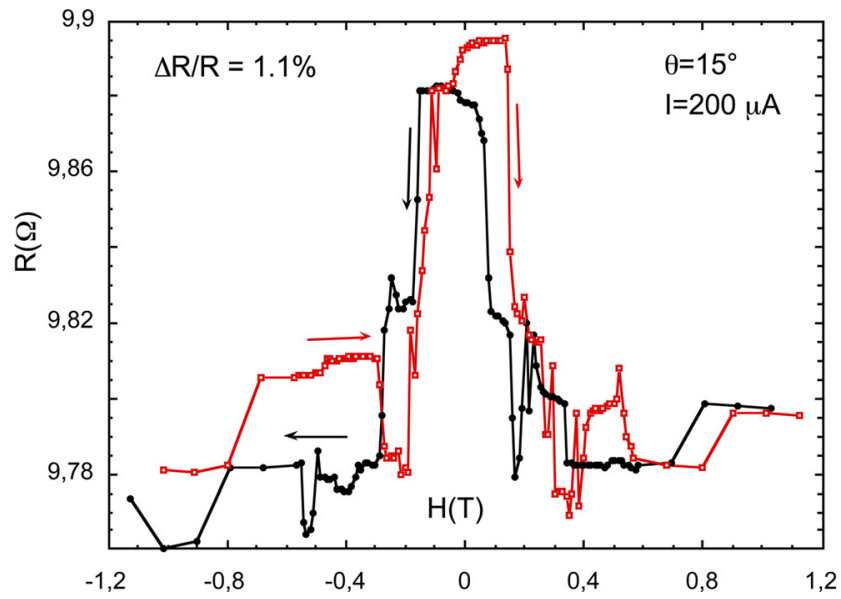

b)

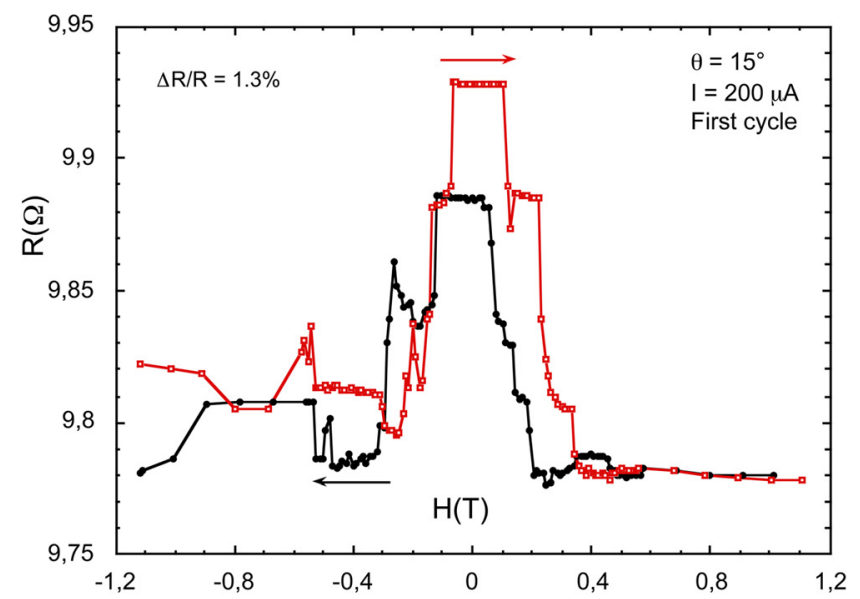

d)

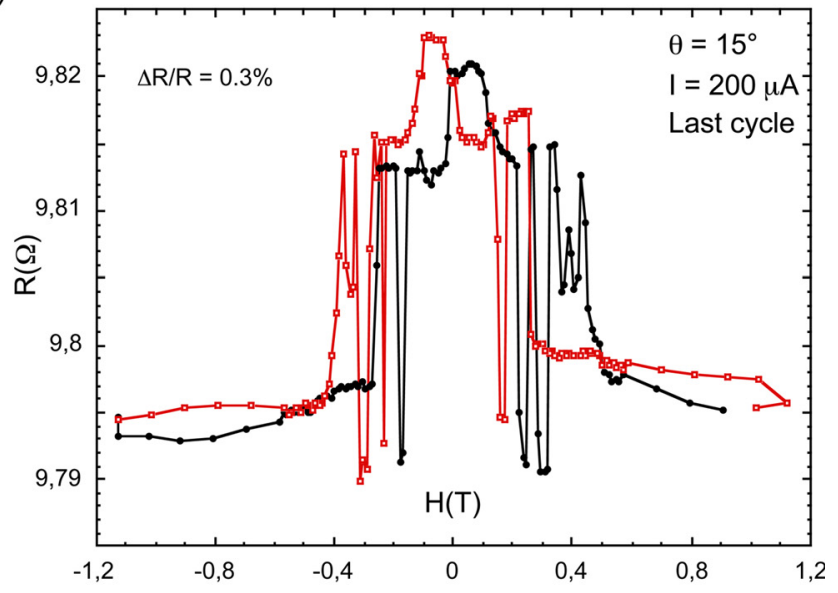

FIG. 5. (a) Time dependence of the resistance measured during ten successive hysteresis loops $( \pm 1.2 \mathrm{~T})$ at an angle $\theta=15^{\circ}$. Hysteresis loops plotted as a function of the magnetic field, (b) $t \approx 0 \mathrm{~s}$, (c) zoom for $t \approx 500 \mathrm{~s}$, and (d) zoom for $t \approx 1500 \mathrm{~s}$ with unstable states.

\section{DISCUSSION}

Even if we suppose the existence of a complicated magnetic configuration with unusual signals, it would be difficult to interpret the hysteresis loops presented in Figs. 3(b) and 4 on the basis of the AMR properties. Indeed, let us suppose a contrario that the $R(H)$ profile is due to AMR (i.e., that Eq. (1) is valid). Since at $\theta=90^{\circ}$ and high fields $( \pm 1.2 \mathrm{~T})$ the resistance is not at the minimum, it is necessarily dominated by the contribution of a pinned Domain Wall (DW) that does not align with the magnetic field. But if the DW is still present despite the high fields, it would be difficult to understand why the resistance decreases at low field, as shown in Fig. 3(b). Accordingly, the hypothesis an AMR profile due to displacement of a DW under the magnetic field is difficult to admit.

This is the reason why we think that the observed signal is caused by a magnetomechanical field of strain generated by the magnetostriction of the nickel. The action of the strain at the level of the constriction leads to plastic deformations. Few Angstrom deformations are sufficient to close or open a conducting channel of about $13 \mathrm{k} \Omega$ resistance. Since only a limiting amount of conducting channels are available in the constriction (typically in the range of 10 to 100 channels contacted in parallel) and taking into account that a bunch of ten bicones are contacted in parallel, the resulting change of resistance would range between $1 \%$ and $0.1 \%$, in agreement with the measurements.

Due to the magnetostriction properties, the volume of the Ni material follows an hysteresis loop which is closely related to the magnetic hysteresis loop, ${ }^{7}$ and the resulting strain follows also approximately the same profile, as it is observed on the magnetoresistance. Note that the typical features measured here have already been observed in other types of constrictions obtained in track-etched nanopores. ${ }^{8}$ It is however difficult to relate the magnetic configurations to the strain exerted on the constriction via the magnetostriction formula, ${ }^{7}$ because it would need to know the geometry of the constriction and the role of the membrane in the transmission of the strain.

This scenario is corroborated by the slow relaxation effects systematically measured with the change of magnetoresistance. The relaxation can be understood on the basis of the change of the lattice structure at the level of the constriction. The effect of the magnetomechanical strain would be to induce the displacement of dislocations or similar structural defects. Thermal activation or cycling with the external magnetic field allows for the system to relax slowly to more 

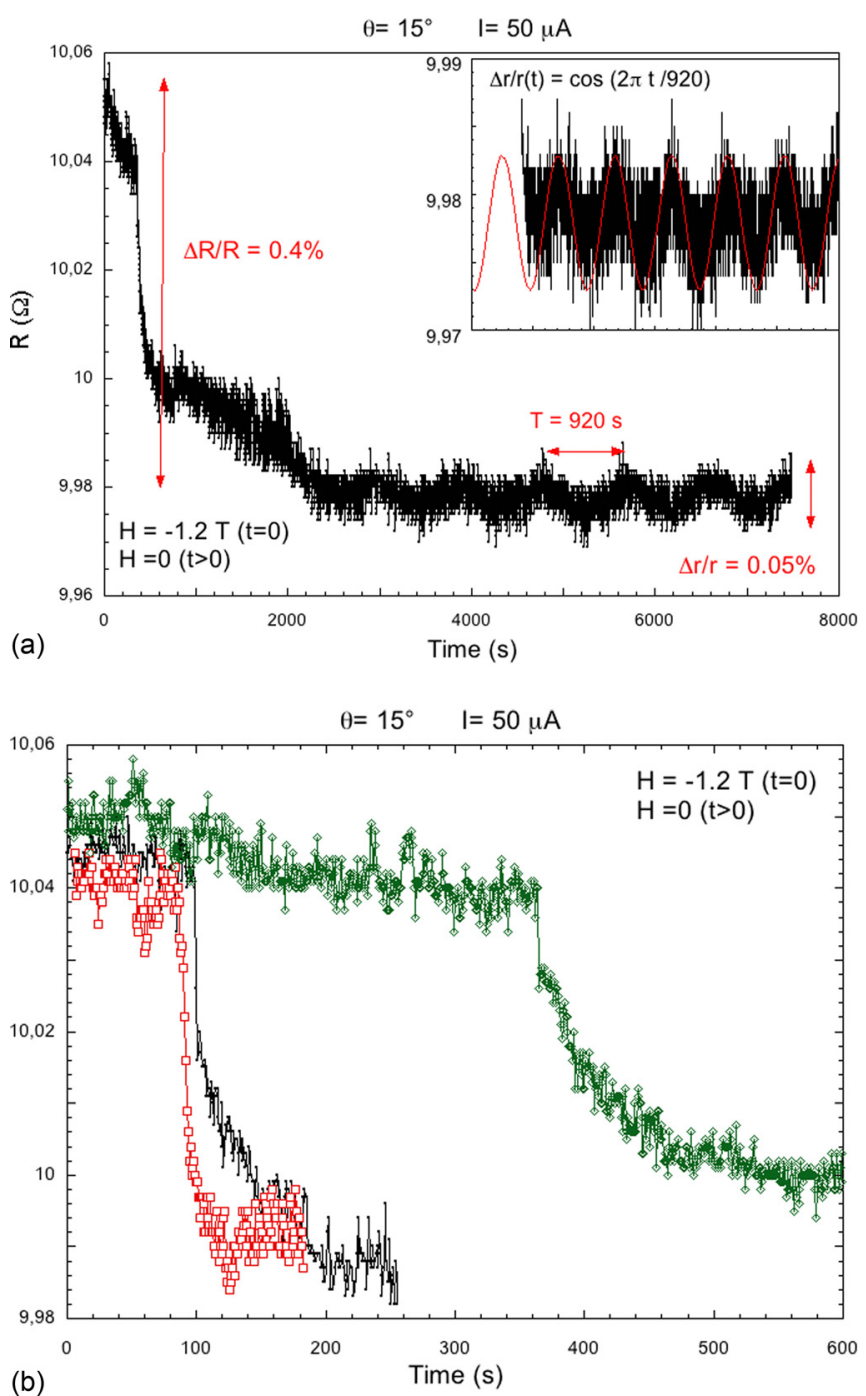

FIG. 6. (a) Time dependence of the magnetization (magnetic after-effect) at small angle $\left(\theta=15^{\circ}\right)$. At time $\mathrm{t}=0$, the magnetic field is swept from $\mathrm{H}=-1.2 \mathrm{~T}$ to $\mathrm{H}=0 \mathrm{~T}$. Different relaxation mechanisms take place. Inset: zoom on the undamped oscillations with the cosine fit. (b) Relaxation time is very sensitive to the magnetization state, which varies slightly from one relaxation measurement to the other.

stable mechanical states defined by the distribution of pinning centers for an ensemble of dislocations.

The undamped oscillations observed can then be interpreted as the consequence of a two level fluctuation effect, thermally activated over a double well potential. The corresponding relaxation time is given by the period $\mathrm{T}$ measured in Fig. 6(a), which corresponds to an energy barrier $\Delta E$ of the order of $\Delta E / k T=25.3$. Note that the relaxation processes between two well defined positions (i.e., two minima of the potential energy) of a dislocation; typically in the case of the creation and annihilation of a single metastable kink, the relaxation curve (Fig. 6(a)) should be a two level fluctuation process-or telegraph noise-composed of a succession of instantaneous jumps of identical amplitude, instead of an oscillation like that observed. However, the signal to noise ratio is too small to allow the two levels to be well separated, and the displacements of a couple of connected kinks in a dislocation line contains more than two levels. In conclusion, the ensemble of observations is compatible with the displacement of dislocations in the region of the constriction.

\section{CONCLUSION}

The study of the magnetoresistance produced in a bi-conical Ni nanowire with a small constriction shows some typical features that cannot be interpreted by usual magnetoresistance properties. The magnetoresistance signal is inverted with respect to the expected and well-known anisotropic magnetoresistance profile. Irreversible resistance jumps are observed with systematic slow relaxation effects. The jumps are separated by stable plateaus. We interpret these features by the action of a field of strain produced by the magnetostriction of the Ni bicones. This field of strain acts significantly at the level of the nanoconstriction with displacing structural defects (typically dislocations originally present or produced by strain). Assuming that the constriction is composed of a dozen of parallel ballistic channels, a displacement of a few Angstroms is able to account for the observed dramatic changes of resistance. The displacement leads hence to a plastic deformation that opens or closes conducting channels of about $13 \mathrm{k} \Omega$ each.

\section{ACKNOWLEDGMENTS}

This work was supported by the RTRA program Triangle de la Physique, Project 2011085 T DECELER and by Direction Générale de lArmement (DGA, France) REI n08C0035.

${ }^{1}$ P. Bruno, Phys. Rev. Lett. 83, 2425 (1999).

${ }^{2}$ P. O. Jubert and R. Allenspach, J. Magn. Magn. Mat. 290, 758 (2005).

${ }^{3}$ M. Viret, D. Vignoles, D. Cole, J. M. D. Coey, W. Allen,D. S. Daniel, and J. F. Gregg, Phys. Rev. B 53, 8464 (1996).

${ }^{4}$ L. Berger and J. Berger, J. Appl. Phys. 49, 2156 (1978).

${ }^{5}$ A. Thiaville, Y. Nakatani, J. Miltat, Y. Suzuki, Europhys. Lett. 69, 990-996 (2005).

${ }^{6}$ B. Doudin and M. Viret, J. Phys. Condens. Matter 20, 083201 (2008).

${ }^{7}$ Magnetism, Fundamentals, Joseph Fourier ed., edited by E. du Tremolet de Lacheisserie, D. Gignoux, and M. Sclenker (2002), Chap. 12, Sec. 4.5 (typically Fig. 12.22).

${ }^{8}$ J.-E. Wegrowe, T. Wade, X. Hoffer, L. Gravier, J.-M. Bonard, and J. Ph. Ansermet, Phys. Rev. B 67, 104418 (2003).

${ }^{9}$ M. Gabureac, F. Ott, C. Fermon, and M. Viret, Phys. Rev. B 69, 100401 (2004).

${ }^{10}$ W. F. Egelhoff, Jr., L. Gan, H. Ettedgui, Y. Kadmon, C. J. Powell, P. J. Chen, A. J. Shapiro, R. D. McMichael, J. J. Mallett, T. P. Moffat, M. D. Stiles, and E. B. Svedberg, J. Magn. Magn. Mater. 287, 496 (2005).

${ }^{11}$ N. Bizière, R. Lassalle Ballier, M.-C. Clochard, M. Viret, T. L. Wade, and J.-E. Wegrowe, J. Appl. Phys. 110, 063906 (2011).

${ }^{12}$ M -C. Clochard, M. El Jouad, N. Bizière, P. Do Chung, H.-J. Drouhin, E. Balanzat, D. Lairez, M. Viret, and J.-E. Wegrowe. "Magnetic nanoconstrictions made from nickel electrodeposition in polymeric bi-conical tracks: Magneto-transport behaviour," Radiat. Phys. Chem. (submitted).

${ }^{13}$ M.-C. Clochard, T. L. Wade, J.-E. Wegrowe, and E. Balanzat, Nucl. Instrum. Methods Phys. Res. B 265, 325-329 (2007).

${ }^{14}$ C. Tasserit, A. Koutsioubas, D. Lairez, G. Zalczer, and M.-C. Clochard, Phys. Rev. Lett. 105, 260602 (2010).

${ }^{15}$ T. L. Wade and J.-E. Wegrowe, Eur. Phys. J.: Appl. Phys. 29(1), 3-22 (2005).

${ }^{16}$ J.-E. Wegrowe, D. Kelly, A. Franck, S. E. Gilbert, and J.-Ph. Ansermet, Phys. Rev. Lett. 82, 3681 (1999).

${ }^{17}$ T. R. McGuire and R. I. Potter, IEEE Trans. Magn. 11, 1018 (1975). 